\title{
Synchronous Diagnosis of Primary Myelofibrosis and Chronic Lymphocytic Leukemia: A Case Report and Literature Review
}

\author{
Pier Paolo Piccaluga ${ }^{1-3}$, Carlo Sagramoso Sacchetti ${ }^{1}$, Serah Kaggia $^{3}$ and Giuseppe Visani ${ }^{4}$ \\ ${ }^{1}$ Department of Experimental, Diagnostic, and Specialty Medicine, Italy \\ ${ }^{2}$ Istituto Euro-Mediterraneo di Scienza e Tecnologia (IEMEST) Palermo, Italy \\ ${ }^{3}$ Department of Pathology, Kenya \\ ${ }^{4}$ Hematology and Stem Cell Transplantation, Italy
}

*Corresponding author: Pier Paolo Piccaluga, Department of Experimental, Diagnostic, and Specialty Medicine, Italy

ARTICLE INFO

Received: 崖 May 16, 2019

Published: May 23, 2019

Citation: Pier Paolo P, Carlo Sagramoso S, Serah K, Giuseppe V. Synchronous Diagnosis of Primary Myelofibrosis and Chronic Lymphocytic Leukemia: A Case Report and Literature Review. Biomed J Sci \& Tech Res 18(2)-2019. BJSTR. MS.ID.003128.

\begin{abstract}
The association between lymphoproliferative disorders and myeloproliferative neoplasms is well recognized. However, only few cases have been described so far of Chronic Lymphocytic Leukemia (CLL) simultaneously diagnosed with Primary Myelofibrosis (PMF). We describe a new case of CLL-PMF association and briefly review the other cases reported in literature. A 79 years-old female was diagnosed for PMF based on clinical features, peripheral blood counts and cytology as well as on bone marrow histological examination. At the same time, based on peripheral blood lymphocytes count and immunophenotype (evaluated at flow cytometry) and bone marrow features, a diagnosis of CLL was made. Despite uncommon, the association between the two disease is possible and worthy of consideration. The molecular bases of the phenomenon need to be assessed as well as the clinical consequences.
\end{abstract}

Keywords: Primary Myelofibrosis; Chronic Lymphocytic Leukemia; Bone Marrow Biopsy; Chronic Myeloproliferative Neoplasm; Immunohistochemistry

\section{Mini-Review}

Chronic Lymphocytic Leukemia/Lymphoma (CLL) can be associated with several malignancies, but rarely with Myeloproliferative Neoplasms (MPN), including Myelofibrosis (MF). Few studies as well as isolated case reports described the association between CLL and idiopathic MF in the same patient [17]. A recent, comprehensive review of literature identified described 49 patients affected by MF that developed a lymphoproliferative disorder [8]. Among them, only 23 had CLL and in 7 instances only the diagnoses were synchronous [8].

Herewith, we present the bone marrow appearance of a 79 years-old female presenting with fatigue, enlarged spleen at physical examination, and peripheral blood abnormalities (WBC, 24x109/L; lymphocytes, 22x109/L; Hb, 11,6 g/dl; PLT, 96x109/L; evidence of anisocytosis, poykylocytosis, as well as presence of dacryocytes at peripheral blood morphological examination).
At low magnification, cellularity appeared highly increased, being roughly 100\% (Figure 1A). A marked granulopoiesis expansion was observed, with increase of proliferating elements; mature hematopoietic cells were, nonetheless, present (Figure 1B). Megakaryocytes (MKC) were evidently expanded and constituted by dystrophic elements, variable in size (from small to big) and focal tendency to clustering (Figure 1C-D; please note the yellow arrow indicating MKC clustering). Reticulin fiber deposition was only mildly increases (Figure 1E). Molecular data were not available. Based on the combination of clinical and pathological evidences, a diagnosis of primary myelofibrosis, early phase, was made.

In addition, however, within the packed hematopoietic elements, some nodular infiltrated were noted. The latter were constituted by small-sized lymphocytes with hyperchromatic 
nuclei and larger elements with more pronounced nuclei and evident nucleolus corresponding to prolymphocytes (yellow arrow) and para-immunobolasts (red arrows) (Figure 2A). At immunohistochemistry, the lymphoid components (both smaller and larger) turned out to be CD79A-positive (Figure 2B), CD5- positive and CD23-positive. The same typical phenotypic pattern was observed in the peripheral blood lymphocytes at flow cytometry (CD19+/CD5+/CD23+). Based on that, a diagnosis of concomitant CLL was made. The patient was under wait and watch at the time of writing.

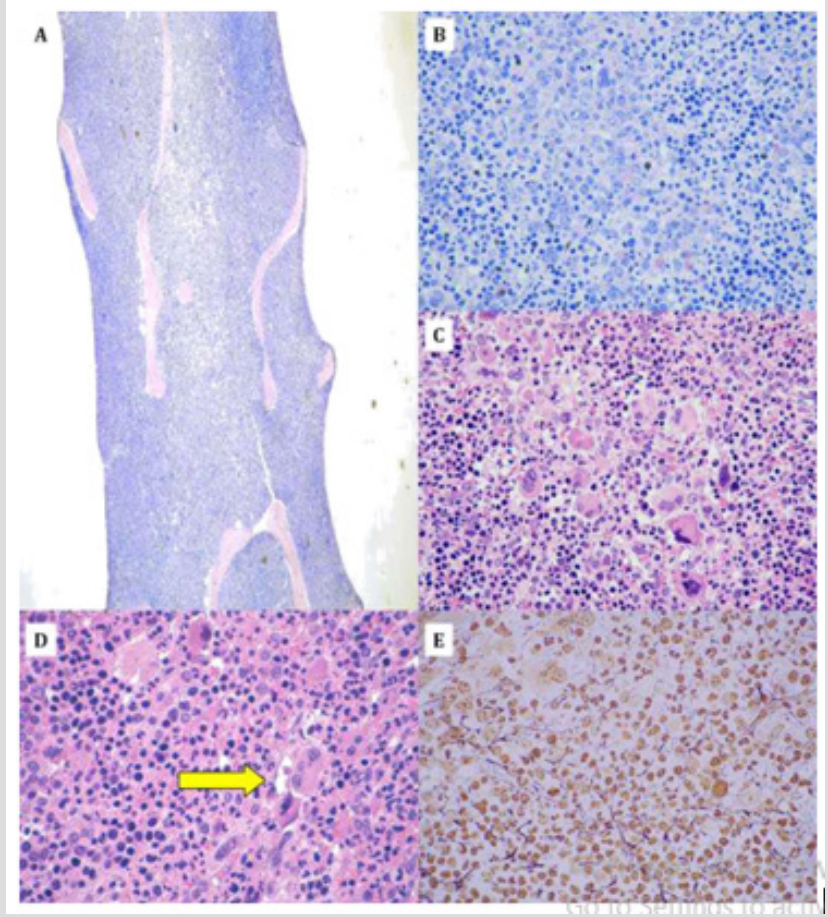

Figure 1: Evidence of myelofibrosis at bone marrow trephine biopsy. Increased cellularity, close to $100 \%$, at low magnification (Figure 1A; 10x, Olympus BX40, GIEMSA staining). Marked granulopoiesis expansion, with increase of proliferating elements and residual mature hematopoietic cells were (Figure 1B; 40x, Olympus BX40, GIEMSA staining). Megakaryocytes (MKC) were evidently expanded and constituted by dystrophic elements, variable in size (from small to big) and focal tendency to clustering (Figure 1C-D; please note the yellow arrow indicating MKC clustering. 40x, Olympus BX40, Hematoxylins \& Eosin staining). Reticulin fiber deposition was only mildly increases (Figure 1E; 40x, Olympus BX40, Silver staining).

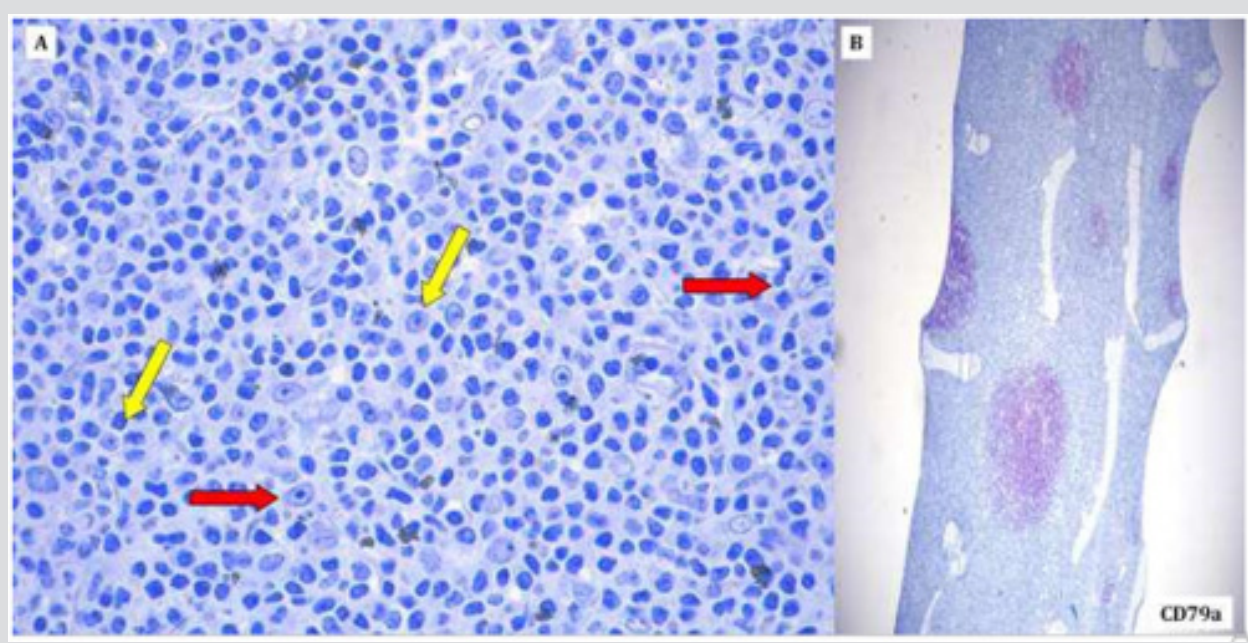

Figure 2: Evidence of chronic lymphocytic leukemia within the bone marrow. Nodular structurers within the packed hematopoietic elements were constituted by small-sized lymphocytes with hyperchromatic nuclei and larger elements with more pronounced nuclei and evident nucleolus corresponding to prolymphocytes (yellow arrow) and para-immunobolasts (red arrows) (Figure 2A; 40x, Olympus BX40, GIEMSA staining). At immunohistochemistry, the lymphoid components (both smaller and larger) turned out to be CD79A-positive (Figure 2B; 10x, Olympus BX40, GIEMSA counter-staining). 
A recent comprehensive review identified $50 \mathrm{MF}$ cases that occurred in patients also diagnosed for a lymphoproliferative disease, this being CLL in 23 cases [8]. Specifically, CLL diagnosis preceded the MF one in 12 cases, followed in 4, while the two diagnoses were synchronous on 7 patients [8]. So far, however, no clear correlation has been established between the genetic feature of these patients (including possible single nucleotide polymorphisms favoring both tumors) and the occurrence of the malignancies. Similarly, how the presence of a second cancer did affect patients' outcome is still largely undefined.

\section{Conclusion}

In conclusion, this case confirmed the possible rare association between MF and CLL and highlights the importance of an accurate diagnostic work up. Future studies should focus on the molecular basis of the phenomenon and try to establish how the second cancer affects the outcome of these patients.

\section{References}

1. Lennard AL, Proctor SJ (1984) Chronic lymphocytic leukemia terminating in acute myelofibrosis. J Clin Patho 37: 564-567.

2. Nieto LH, Raya Sánchez JM, Arguelles HA, Brito Barroso ML, González BG (2000) A case of chronic lymphocytic leukemia overwhelmed by rapidly progressing idiopathic myelofibrosis. Haematologica 85: 973-977.

\section{ISSN: 2574-1241}

DOI: 10.26717/BJSTR.2019.18.003128

Pier Paolo Piccaluga. Biomed J Sci \& Tech Res

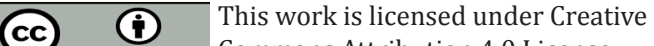

Submission Link: https://biomedres.us/submit-manuscript.php
3. Palta A, Garg S, Chauhan S, Varma N (2011) Simultaneous presence of two hematological malignancies: Chronic lymphocytic leukemia and myelofibrosis in a patient. Indian J Hematol Blood Transfuse 27: 33-34.

4. Burgstaller S, Wimmer S, Mayrbaeurl B, Hoebling W, Thaler J (2011) Coexistence of primary myelofibrosis and chronic lymphocytic leukemia: Treatment of two different diseases with one agent. Blood Cancer Journal 1: e20.

5. Laurenti L, Tarnani M, Nichele I, Ciolli S, Cortelezzi A, et al. (2011) The coexistence of chronic lymphocytic leukemia and myeloproliferative neoplasms: A retrospective multicentric GIMEMA experience. Am J Hematol 86: 1007-1012.

6. Kimura A, Hyodo H, Nakata Y, Kuramoto A (1993) Chronic lymphocytic leukemia associated with bone marrow fibrosis: Possible role of interleukin 1 alpha in the pathogenesis. American Journal of Hematology 43: 47-50.

7. Marchetti M, Carobbio A, Capitoni E, Barbui T (2018) Lymphoproliferative disorders in patients with chronic myeloproliferative neoplasms: A systematic review. Am J Hematol 93(5): 698-703.

8. Todisco G, Manshouri T, Verstovsek S, Masarova L, Pierce SA, et al. (2016) Chronic lymphocytic leukemia and myeloproliferative neoplasms concurrently diagnosed: Clinical and biological characteristics. Leuk Lymphoma 57(5): 1054-1059.

$\begin{array}{ll}\text { BIOMEDICAL } & \text { Assets of Publishing with us } \\ \text { RESEARCHES } & \text { - Global archiving of articles } \\ & \text { - Immediate, unrestricted online access } \\ & \text { - Rigorous Peer Review Process } \\ \end{array}$

